\title{
An average model for disordered sphere packings
}

\author{
Calixtro Yanqui ${ }^{{ }^{*}}$ \\ ${ }^{1}$ Civil Engineering Department, National University of Saint Agustine, Arequipa, Peru
}

\begin{abstract}
In this paper, an assembly of disordered packings is considered as a suitable set of packing cells of ordered spheres. In consequence, any of its parameters can be obtained by averaging the values of the set. Namely, the density of a packing of ordered spheres is described by two variables: the angle of the base, and the angle of the inclined edge of the associated parallelepiped. Then, the density of a packing of disordered spheres is obtained by averaging the angle of the base, and the subsequent averaging of the other angle, according to the kind of strain induced by the experiment. The average packing yields the density limits of loose sphere assemblies achieved by a process of fluidization and sedimentation in air, in water, and in viscous liquid at zero gravitational force. It also models the close sphere assemblies shaped by gentle tapping, vertical shaking, horizontal and multidirectional vibrations. The theory allows to elucidate the mechanism of each of the limits, as, for example, the metastable columns of spheres in the loosest packing, as well as the random close packing, and crystallization. The limits obtained coincide very well with the published experimental, numerical and theoretical data.
\end{abstract}

\section{Introduction}

Granular materials have complex mechanical behaviour as a consequence of the complicated interaction between grains. To simplify the picture, it has been proposed to replace the assemblages of grains with packings of spheres. In engineering, as a first condition, the model should serve to explain and describe the complete strain history of the samples in the simple shear and triaxial tests. As a second condition, the model should allow to solve complicated field problems of stress distribution, strain, and failure. To meet this objectives, the model should be as simple as possible. That is the disorderly and deformable packing of rigid spheres, the rearrangement of which results in the change of shape of the packing. For this model, one of the main tasks is the determination of the extreme packings, associated to the loosest and densest states. Another problem is the modelling of the strain response, whose most important component is the displacement of the spheres.

The extreme states of disordered packings of hard spheres have received the attention of many authors, since the pioneering experiments of Bernal and Mason [1] and Scott [2]. The density of the loose state has been refined from 0.608 [2], 0.570 [3] down to 0.550 [4]. Similarly, the density of the close packings has evolved from 0.620 [2], 0.640 [1], up to 0.700 [5], by means of sophisticated experimental methods, numerical simulations and application of the statistical mechanics.

In this work a new theoretical method is proposed to find the disordered packing densities, based on the averaging of all possible packings defined by the base angle of a particular unit cell of the Bravais triclinic system. Two cells are considered: the rhombohedron with a horizontal face or prismatic packing, subject to shear deformation; and the parallelepiped defined by a pyramid with an isosceles triangular base, subject to triaxial compression. The model is strictly geometric and does not involve the effect of the friction angle. The relationship between this parameter and the density of soils has been studied empirically and theoretically by several authors [6]. Paraphrasing Tolman [7], the proposed method is to be regarded as really statistical in character, and the results which they provide are to be regarded as true on the average for the systems in an appropriately chosen set, rather than as necessarily precisely true in any individual case.

\section{Packings of ordered spheres}

The geometry of ordered packings obeys the laws of lattices or crystalline arrangements, and the volume of their phases can be easily calculated, since it is always possible to select a parallelepiped, whose vertices are the center of a sphere (Fig. 1a). In this primitive unit cell, the spheres of the base are not necessarily in contact, and the spheres of two successive layers are in contact at the middle point of the inclined edge. The total volume of the parallelepiped is found to be: $V=a b c \sin \alpha \cos \varsigma$, where $a, b$, and $c$ are the lengths of the edges; $\alpha$, the angle of the base; and $\zeta$, the angle of the inclined edge to the vertical axis (Fig. 1b). The angle $\alpha$ defines the geometry of the base, and varies within the interval: $60^{\circ} \leq \alpha \leq 90^{\circ}$. The first limit value corresponds to the hexagonal monoclinic system, and the second, to the quadratic monoclinic system.

The volume of solids is a constant, since the oblique octants subtended by the faces of the parallelepiped are

* Corresponding author: cyanqui@unsa.edu.pe

A video is available at https://doi.org/10.48448/1hp2-6959 
complementary and so are the portions of the spheres at each vertex. The sum of them is equal to the volume of a solid sphere of diameter $D: V_{\mathrm{s}}=\pi D^{3} / 6$, independently of the angles $\alpha$ and $\zeta$.

Then, the density of the packing is given by the relation $\phi=V_{\mathrm{s}} / V$. However, as will be seen later, a more useful parameter is the specific volume, $v$, defined in the theory of the critical state of soils as the inverse of the density of the packing:

$$
v=\frac{1}{\phi}=\frac{6 a b c}{\pi D^{3}} \sin \alpha \cos \varsigma
$$
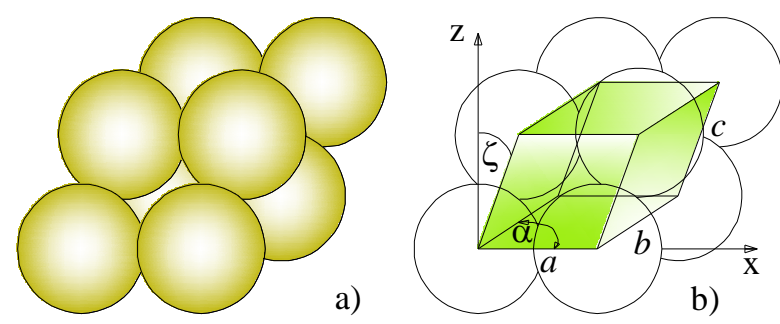

Fig. 1. Geometry of the ordered packing of spheres: a) volumetric view, b) parameters of the basic parallelepiped

In a prismatic packing, the contact of the spheres requires that: $a=b=c=D$, and $\varsigma=\beta$, where $\beta$ is the angle of shear deformation. Accordingly, the specific volume is given by the equation:

$$
v=\frac{6}{\pi} \sin \alpha \cos \beta
$$

In a pyramidal packing, the edge lengths of the triangular pyramid are $a=b=2 D \cos (\alpha / 2) \sin \theta$, and $c=D$. The angle $\varsigma$ has different meaning, and is substituted by the axial angle $\theta$, that is related to the Poisson's effect. Then, the specific volume is found to be:

$$
v=\frac{12}{\pi}(1+\cos \alpha) \sin \alpha \sin ^{2} \theta \cos \theta
$$

\section{Extreme states of ordered packings}

\subsection{Prismatic packing}

The loosest state of the packing is obtained by maximizing the equation (2) with respect to the angle of the inclined edge, and with respect to the angle of the base of the parallelepiped. Those are: $\beta=0^{\circ}$ and $\alpha=90^{\circ}$. For these values, the density of the packing is $\phi=1 / v=\pi / 6=0.524$. However, saving only the $\operatorname{root} \beta=0^{\circ}$, there is another extreme angle of the base: $\alpha=60^{\circ}$, since $60^{\circ} \leq \alpha \leq 90^{\circ}$. For this value, the density of the packing is $\phi=1 / v=\pi / 3 / \sqrt{ } 3=0.605$. It is important to note that the structure of the packings under the condition $\beta=0^{\circ}$ are made of columns of spheres, which generate a metastable system.

The dense state is achieved when the sphere of the upper layer comes into contact with the three spheres of the isosceles triangle of the base. The extreme states are obtained when the angle of the base reaches the values $\alpha=60^{\circ}$ and $\alpha=90^{\circ}$, giving a single density for the two packings: $\phi=\pi \sqrt{ } 2 / 6=0.740$. For the tetrahedral packing the angle of distortion is $\beta_{1}=\sin ^{-1}(1 / \sqrt{ } 3)=35.26^{\circ}$, and, for the octahedral packing: $\beta_{2}=45^{\circ}$. It is worth to note that the value $\phi=0.740$ is valid only for these two extreme prismatic packings.

\subsection{Pyramidal packing}

The loose state of a pyramidal packing is achieved by differentiating the specific volume with respect to the angle of the inclined edge, which yields: $\theta=\cos ^{-1}(1 / \sqrt{ } 3)$ $=54.74^{\circ}$. The densities of the extreme packings with respect to the angle of the base are: (a) tetrahedral packing: $\alpha=60^{\circ} ; \phi=1 / v=\pi / 6=0.524$, (b) octahedral packing: $\alpha=90^{\circ} ; \phi=1 / v=3 \pi / 8 / \sqrt{ } 3=0.680$. This is a stable system since each sphere of the upper layer rests on three spheres of the lower layer, leading to an isostatic equilibrium, except for the octahedral packing, which has four contacts, becoming a hyperstatic system.

The dense state of the pyramidal packing is obtained by increasing the number of contacts among the spheres of the base. This makes the pyramidal packing equal to the prismatic packing, for this case. Therefore, the packing density for the densest state is $\phi=\pi \sqrt{ } 2 / 6=0.740$, for the tetrahedral packing, whose axial angle is given by $\theta_{2}=\sin ^{-1}(1 / \sqrt{ } 3)=35.26^{\circ}$, and for the octahedral packing, whose axial angle is given by $\theta_{1}=45^{\circ}$.

\section{Extreme states of average packings}

The simplest way to find the density of a packing of disordered monodisperse hard spheres is averaging the densities of all possible ordered packings related to the kind of experiment. To obtain better results, the averaging is performed with respect to angle $\alpha$, saving the angle of the inclined edge as a physical or statistical parameter of the experiment.

\subsection{Prismatic packing}

Since the interval of validity of the angle of the base is $60^{\circ} \leq \alpha \leq 90^{\circ}$, the specific volume of the prismatic average packing is:

$$
v=\frac{36}{\pi^{2}} \cos \beta \int_{\pi / 3}^{\pi / 2} \sin \alpha d \alpha=\frac{18}{\pi^{2}} \cos \beta
$$

a) The density of the loosest prismatic packing is found by knowing that this state occurs when $\beta=0$. So, the minimum density is given by: $\phi=\pi^{2} / 18=0.548$. Since the columns of spheres remain despite averaging, this is a metastable system.

b) The density of the closest packing is not given by a single value of $\beta$, but by an interval whose limits are defined by the tetrahedral and octahedral packings. Therefore, it is necessary to average the specific volume, $v$, by integrating the function (4) between the limits $\beta_{1}=\sin ^{-1}(1 / \sqrt{ } 3)=35.26^{\circ}$, and $\beta_{2}=45^{\circ}$, of the crystalline 
packings, and dividing by the interval $\Delta \beta=\beta_{2}-\beta_{1}$. That is: $\phi=\pi^{2} \sqrt{6} 6(\sqrt{3}+\sqrt{ } 2) / 18 \sin ^{-1}((\sqrt{2}-1) / \sqrt{ } 6)=0.718$.

c) The minimum density of a close packing, which is related to the phase change, is given by the lower limit of the close states: $\beta_{1}=\sin ^{-1}(1 / \sqrt{ } 3)=35.26^{\circ}$. The result is $\phi=\pi 2 \sqrt{6} 6 / 36=0.671$.

d) The density of an average packing of totally disordered spheres is obtained by integrating the specific volume from $\beta_{1}=0$ to $\beta_{2}=45^{\circ}$, from the loose state to the close state, and dividing by the interval $\Delta \beta=\beta_{2}-\beta_{1}=\pi / 4$. The result is: $\phi=\pi^{3} \sqrt{2} / 72=0.609$. This value has been considered by several authors as the density of the random loose packing (RLP).

\subsection{Pyramidal packing}

The stress-strain curve of the triaxial compression depends on the axial angle, $\theta$, so that the angle of the base of the parallelepiped, $\alpha$, is the parameter to be integrated to find the average specific volume:

$$
v=\frac{36}{\pi^{2}} \sin ^{2} \theta \cos \theta \int_{\pi / 3}^{\pi / 2}(1+\cos \alpha) \sin \alpha d \alpha=\frac{45}{\pi^{2}} \sin ^{2} \theta \cos \theta
$$

a) The loosest state of the average pyramidal packing is obtained maximizing function (5). This operation leads to: $\theta_{0}=\cos ^{-1}(1 / \sqrt{ } 3)=54.74^{\circ}$. Then, the density of the packing is: $\phi=\pi^{2} / \sqrt{ } 3 / 10=0.570$. Since a sphere of the upper layer rests on three spheres of the lower layer, this is a stable loose packing.

b) The density of the closest average packing is obtained by integrating the function (5) from $\theta_{1}=45^{\circ}$ to $\theta_{2}=35.26^{\circ}$ and dividing by the interval $\Delta \theta=\theta_{2}-\theta_{1}$. That is: $\phi=2 \pi^{2} \sqrt{6}(3 \sqrt{3}+2 \sqrt{2}) / 95 \sin ^{-1}((\sqrt{ } 2-1) / \sqrt{ } 6)=0.694$.

c) The minimum density of close packings is giving by the upper limit of the angle of the inclined edge of the basic parallelepiped: $\theta=45^{\circ}$. Substituting this value in equation (5), it is found: $\phi=2 \pi^{2} \sqrt{ } 2 / 45=0.620$.

d) The density of a totally disordered pyramidal packing is achieved integrating the function (5) over the total interval of the angle $\theta$; from $\theta_{0}=\cos ^{-1}(1 / \sqrt{ } 3)$ $=54.736^{\circ}$; to $\theta_{2}=\sin ^{-1}(1 / \sqrt{3})=35.26^{\circ}$ and dividing by $\Delta \theta=\theta_{2}-\theta_{0}$. This equation leads to the following packing density: $\phi=\pi^{2} \sqrt{3} \sin ^{-1}(1 / 3) / 5 /(2 \sqrt{2}-1)=0.635$. This value corresponds to the random close packing (RCP)

\section{Evaluation of results}

Determining the density of disordered assemblages of hard spheres has been the subject of various branches of physics, chemistry and engineering. In particular, the limit densities have been studied by several authors, using experimental, numerical and analytical methods.

\subsection{Loose packings poured in air}

Scott [2], among others, prepared samples of steel balls, gently pouring them into a container, finding the following packing density: $\phi=0.608$. Schröter et al. [8] determined a critical density of $\phi=0.590$, using a different method, which consisted of extracting a rod from the assembly contained in a cylinder. Song et al. [9] found for a pyramid with a square base (coordination number 5) the following density $\phi=0.591$.

These values are close to the density of $\phi=0.609$, obtained for an average prismatic packing of totally disordered spheres. Interestingly, a similar density is obtained for the crystalline hexagonal packing.

\subsection{Fluidization and sedimentation in water}

Brown and Hawksley [3] reported a value of 0.570 for loose packing of steel ball bearings poured rapidly into a rather small cubical container. The experiment of Schröter et al. [8] consisted of subjecting a layer of fluidized balls in water to a series of flow pulses and then allowing them to settle. The result was $\phi=0.571$. These experiments improve the first tests on the density of loose packings. This value coincides with that obtained for the loosest average tetrahedral packing, whose density is $\phi=0.570$, which perfectly matches the experimental data.

\subsection{Fluidization and sedimentation at zero gravitational force}

Under extreme fluidization and sedimentation conditions, Onoda and Lininger [4] have achieved packing densities as low as $\phi=0.555$. To do that, the spheres have been fluidized in a liquid whose density is slightly less than the density of the spheres and have been settled in an almost zero gravitational field. Song et al. [9] determined a density of $\phi=0.536$ using the statistical mechanics of jamming for a tetrahedral packing (coordination number 4 ).

These values are quite close to the density of the loosest average packing, made up of columns of spheres, whose magnitude is $\phi=0.548$.

\subsection{Close packings by gentle vertical tapping}

Tariot et al. [10] achieved an asymptotic packing density of $\phi=0.620$ using the vertical fluidization submitted to a continuous or bursting upward viscous flow. Ribiere et al. [11] found $\phi=0.620$, for the stationary state of a packing subject to gentle mechanical taps. This value coincides with the theoretical minimum density of close pyramidal packing.

\subsection{Shaken and compressed close packings}

Bernal and Mason [1] filled a rubber balloon with spheres of the same size that was then shaken and compressed for a sufficiently long time, obtaining the densest state: $\phi=0.637$. By gently rotating to a vertical position the cylinder containing the spheres arranged in a low density assembly, Scott et al. [2] vibrated the cylinder at a suitable frequency for a few minutes, obtaining a density of $\phi=0.637$. Bernal and Finney [12], obtained an average of $\phi=0.634$ applying the Voronoi polyhedra to Scott's experiment. An et al. [13] 
experimentally obtained the value of $\phi=0.636$ applying vertical vibration to a column of spheres. Scott and Kilgour [14] reported a more precise value of $\phi=0.637$. Nowak et al. [15] achieved densities from $\phi=0.637$ to $\phi=0.647$ for a tall cylindrical tube subject to discrete, vertical shakes. A density of $\phi=0.634$ was found by Song et al, [5], using the statistical mechanics of jamming. Baranau and Tallarek [16], using the Lubacheski - Stillinger algorithm, determined that the density varies between $\phi=0.634$ and $\phi=0.636$.

These values are to be compared with the density of the total average disordered assemblage of the tetrahedral packing of spheres: $\phi=0.635$.

\subsection{Horizontal one-directional shaking}

Pouliquen et al. [5] carried out a test that consisted of continuously pouring glass spheres at different flow rates into a box subjected to horizontal vibrations of different acceleration and amplitude. The result of the process was the partial crystallization of the packing, reaching a density of $\phi=0.670$.

This value is to be compared with the minimum density of the close average prismatic packing: $\phi=0.670$.

\subsection{Cyclic shear and three-dimensional shaking}

Nicolas et al. [17] improved the previous method by considering a box with rotating walls that are transformed into a parallelepiped filled with spheres subject to horizontal shear; through the periodic movement of these walls, obtaining a density of $\phi=0.693$. Li et al. [18] carried out experiments with spheres of the same size subjected to three-dimensional vibration, varying the amplitude and frequency of the vibration, as well as the size of the deposit and the pouring method. For the samples prepared in full from the beginning, the density they obtained was $\phi=0.689$.

These values are to be compared with the density of the closest average pyramidal packing: $\phi=0.694$.

\subsection{Quasi-crystalline close packings}

Pouliquen et al. [5] obtained a density of $\phi=0.700$ pouring the spheres layer by layer in a box subjected to horizontal vibration. $\mathrm{Li}$ et al. [18] carried out tests with samples of spheres placed in layers during the threedimensional vibration of the deposit. The density of the packing thus obtained was $\phi=0.720$, and corrected for the effect of the walls gave $\phi=0.740$. These values are to be compared with the densest average prismatic packing of spheres: $\phi=0.718$.

\section{Synthesis}

Table 1 shows the comparison between the data reported by several authors about the density limits of packings of disordered spheres obtained by different protocols, and the theoretical values calculated from the average packings.
Table 1. Comparison of packing densities.

\begin{tabular}{|l|c|c|}
\hline \multicolumn{1}{|c|}{ Method } & Experiment & $\begin{array}{c}\text { Average } \\
\text { packing }\end{array}$ \\
\hline Pouring & 0.608 & 0.609 \\
Sedimentation in water & 0.571 & 0.570 \\
Sedimentation at zero g & 0.555 & 0.548 \\
\hline Gentle vertical tapping & 0.620 & 0.620 \\
Shaken and compression & 0.637 & 0.635 \\
Horizontal vibration & 0.670 & 0.670 \\
Three-dimensional vibration & 0.693 & 0.694 \\
Vibration - poured by layers & 0.720 & 0.718 \\
\hline
\end{tabular}

\section{Conclusions}

Average packings are the simplest of all models used to describe packings of disordered monodisperse spheres. They are obtained by averaging the angle of the base of the associated parallelepiped. The additional averaging of the angle of the inclined edge of the prismatic and pyramidal packings yields the densities of the loose and close random packings, respectively. The data reported by several authors fit well with the theoretical values.

\section{References}

[1] J.D. Bernal, J. Mason, Nature 188, 910 (1960)

[2] G.B. Scott, Nature 188, 908-909 (1960).

[3] R.L. Brown, P.G.W. Hawskley, Nature. 157, 585 (1946).

[4] G.Y. Onoda, E.G. Lininger, Phys. Rev. Lett. 64, 2727-2730 (1990).

[5] O. Pouliquen, M. Nicolas, P.D. Weidman, Phys. Rev. Lett. APS 79, 3640-3643 (1997)

[6] C. Yanqui, AIP Conf. Proc. 1542, 197-200 (2013)

[7] R.C. Tolman, The principles of statistical mechanics (Oxford University Press, 1938)

[8] M. Schröter, S. Nägle, C, Radin, H.L. Swinney, Eur. Phys. Lett. 78, 44004 (2008)

[9] C. Song, P. Wang, H.A. Makse, Nature Lett. 453, 06981 (2008)

[10] A. Tariot, G. Gauthier, P, Gondret, EPJ Web of Conf. 140, 10003 (2017)

[11] P. Ribiere, P. Richard, P. Philippe, D. Bideau, R. Delannay, Eur. Phys. J. E 22, 249-253 (2007)

[12] J.D. Bernal, J.L Finney, Discuss. Faraday Soc. 43, 62-69 (1967)

[13] X.Z. An, C.X. Li, R.Y. Yang. R.P. Zou, A.B. Yu, Powder Technology 196, 50-55 (2009)

[14] G.D. Scott, D.M Kilgour, J. Phys. D: Appl. Phys. 2, 863 (1969)

[15] E. R. Nowak, J.B. Knight, E. Ben-Naim, H.M. Jaeger, S.R. Nagel, Phys. Rev. E 57 (2) (1998)

[16] V. Baranau, U. Tallare, Soft Matter 10, 3826 (2014)

[17] M. Nicolas, P. Duru, O. Pouliquen, Eur. Phys. J. E 2, 309-314 (2000)

[18] C.X. Li, X.Z. An, R.Y. Yang, R.P.Zou, A.B. Yu, Powder Technology 208, 617-622 (2011) 